\title{
Maraviroc, An Inhibitor of Chemokine Receptor Type 5, Alleviates Neuroinflammatory Response after Cerebral Ischemia/Reperfusion Injury via Regulating JNK Signaling
}

Beilei Chen

Yangzhou University

Pingping Cao

Dalian Medical College: Dalian Medical University

Xin Guo

Dalian Medical College: Dalian Medical University

Xiaobo Li

Yangzhou University

Li Jiang

Yangzhou University

Xiongfeng Lian

Yangzhou University

\section{Xin Chen}

Yangzhou University

Chao Jiang

Yangzhou University

Luhang Tao

Yangzhou University

Hailong Yu ( $\sim$ hailongyu1982tg@163.com )

Northern Jiangsu People's Hospital https://orcid.org/0000-0002-1557-7465

\section{Research Article}

Keywords: cerebral ischemia/reperfusion, Maraviroc, inflammation, microglia, JNK signal pathway.

Posted Date: September 27th, 2021

DOl: https://doi.org/10.21203/rs.3.rs-914711/v1

License: (c) (1) This work is licensed under a Creative Commons Attribution 4.0 International License.

Read Full License 
Page $2 / 20$ 


\section{Abstract}

Neuroinflammation is a key factor that contributes to the secondary injury after cerebral ischemia/reperfusion (Cl/R) injury. Chemokine receptor type 5(CCR5) has shown its pro-inflammatory effects during central nervous system (CNS) diseases. However, the role of CCR5 in $\mathrm{Cl} / \mathrm{R}$ injury is still unclear. In this study, we administered maraviroc (MVC,APEXBIO,UK-427857), a CCR5 antagonist, to the middle cerebral artery occlusion(MCAO) mice. In vivo studies showed that MVC was successively intraperitoneally (i.p.) with different doses $(5,20$, or $50 \mathrm{mg} / \mathrm{kg}$ body weight) for 3 days after mice MCAO. MVC showed its neuroprotective effects in alleviating neurological deficits and infarct volumes after MCAO. The level of apoptosis and inflammation were remarkably decreased by $\mathrm{MVC}$ treatment after $\mathrm{Cl} / \mathrm{R}$ injury. Subsequently, primary microglia were stimulated with different doses of MVC $(0.2,2,20$ or $200 \mathrm{nM})$ for $12 \mathrm{~h}$ after oxygen-glucose deprivation/reoxygenation model (OGD/R) in vitro. MVC significantly increased the viability of primary microglia after (OGD/R). The expression of pro-inflammatory cytokines (IL-1 $\beta$ and IL-6) in microglia were down-regulated by MVC treatment. Mechanistically, MVC also inhibited the secretion of IL-1 $\beta$ and IL- 6 by microglia after OGD stimulation. Furthermore, the key components of NF-KB pathway were measured in vivo and in vitro after MCAO and OGD. MVC significantly inhibited the activity of NF-KB pathway in the above pathological environments. Finally, our data indicated that MVC treatment decreased the activation of JNK signaling pathway after $\mathrm{Cl} / \mathrm{R}$ injury in vivo and in vitro. The JNK activator anisomycin (ANखBeyotime $₫ S C 0132-5 \mathrm{mg}$ ) reversed the neuroprotective effects of MVC, indicating that the JNK pathway is involved in the anti-inflammatory and anti-apoptotic mechanisms of $\mathrm{MVC}$ in $\mathrm{Cl} / \mathrm{R}$ injury. Our data demonstrated that CCR5 inhibition exhibits neuroprotective effects after $\mathrm{Cl} / \mathrm{R}$ injury. MVC, which is widely used for HIV treatment by its anti-virus effect, is a potential drug for the treatment of ischemic stroke in the future clinical trials.

\section{Introdution}

Acute ischemic stroke is the most common cerebrovascular event with high morbidity and mortality[1].. Based on its complex pathophysiological mechanisms, the interaction of various factors, such as inflammatory damage[2, 3]. and blood-brain barrier destruction, [4]. leads to the apoptosis and necrosis of cerebral tissue and cells. However, there is a lack of effective treatments for ischemic stroke. CC chemokine receptor 5 (CCR5), a $\mathrm{G}$ protein-coupled receptor, has the availability to regulate cell activation and migration in response to chemokines[5].. Based on a previously conducted research, CCR5 has been determined to play a vital role in the immune and inflammatory responses by mediating the chemotactic activity in leukocytes[6].. A previous study showed that CCR5 plays a critical role in intestinal ischemia/reperfusion (I/R) injury[7]. and endotoxin-induced lung injury[8].. Nevertheless, the effects of CCR5 antagonist in $\mathrm{Cl} / \mathrm{R}$ remain unknown. This evidence has led to the successful search for CCR5 antagonists as antiretroviral drugs and the approval of maraviroc for both treatment-experienced and treatment $\mathrm{Cl} / \mathrm{R}$. However, whether MVC possesses neuroprotective effects in cerebral I/R injury remains to be understood. Based on its anti-inflammatory capacity[2, 3]., we hypothesized that MVC may have a protective effect in cerebral I/R injury. In this study, we employed a mice middle cerebral artery occlusion 
(MCAO) model to investigate the role of MVC in the treatment of cerebral I/R injury and its potential mechanisms in mice.

\section{Materials And Mmethods}

\section{Animals}

All adult male C57BL/6J (B6) mice (8-10 weeks old, approximately 25-30 g) were purchased from the Comparative Medical Center of Nanjing Medical University (Jiangsu Province, China). As previously described, the mice were housed in a suitable environment.

\section{Grouping and Drug Administration}

Mice were randomized into four groups as follows: I) control vehicle-treated group (Sham group); II) MVCtreated group (MVC group); III) vehicle-treated $\mathrm{Cl} / \mathrm{R}$ group (Cl/R group); and IV) MVC-treated Cl/R group (Cl/R + MVC group). After the induction of MCAO, mice received intraperitoneal injections with either MVC $(5,20$, or $50 \mathrm{mg} / \mathrm{kg}$ body weight) or the vehicle( $10 \%$ dimethyl sulfoxide, $10 \%$ DMSO,Amresco, Solon, $\mathrm{OH}$, USA) daily for 3 consecutive days.

\section{The Middle Cerebral Artery Occlusion(MCAO) model}

The animals were anaesthetized with pentobarbital sodium. The MCAO mouse model was established as previously described[9].. Briefly, a 6/0 monofilament nylon suture (Doccol Corporation, MA, USA) with a heat-rounded tip was inserted into the beginning of the MCA through the internal carotid artery until the ipsilateral blood flow decreased to less than $30 \%$ of the baseline value, as monitored using laser Doppler flowmetry (Perimed Corporation, Stockholm, Sweden). After $60 \mathrm{~min}$ of occlusion, the filament was withdrawn to allow blood reperfusion. In the sham-operated group, the aforementioned procedure was performed, but a filament was not inserted into the MCA. After the operation, we fed the mice jelly or performed an intraperitoneal (i.p.) injection of normal saline to rehydrate. Pre-dissolved MVC or the same volume of saline was administered to each animal by an i.p. injection at $30 \mathrm{~min}, 24 \mathrm{~h}$ and $48 \mathrm{~h}$ after MCAO in a double-blind manner.

\section{Behavioral analysis of neurological deficit score}

Neuro-behavioural function was assessed using the modified neurological severity score (mNSS) and Longa score on day 3 following MCAO by a blinded observer[10].. The mNSS evaluates neurological deficits through motor, reflex, sensory, and balance testing. The score ranges from 0 , indicating no neurologic deficit, to 18 for animals with the most severe impairment. The Longa score is determined as follows: 0 point, no neurological deficit; 1 point, cannot fully extend the contralateral forelimb; 2 points, tail-catching phenomenon while walking (circling to the contralateral side); 3 points, unsteady in the standing position, falling to the contralateral side; and 4 points, no spontaneous walking and decreased consciousness. Scores of 0-2 are classifiedas mild neurological impairment, while scores of 3-4 are 
classified as severe neurological impairment. The evaluators were blinded to the treatment group of mice during the neurobehavioral tests.

\section{Cerebral Infarction Volume Measurement}

After neurological evaluation, mice were sacrificed and histological evaluation of brain tissue was performed. The brain was sectioned into six slices and incubated with $0.2 \%(\mathrm{w} / \mathrm{v}) 2,3,5$ -

triphenyltetrazolium chloride (TTC, Sigma-Aldrich)at $37^{\circ} \mathrm{C}$ for $15 \mathrm{~min}$ to determine the infarct volume. Infarcted tissue was pale grey in color, compared to the dark red color of normal brain tissue. Images were obtained using a digital camera and analyzed using ImageJ software. The percentage of hemispheric infarction volume was calculated using the following formula: Infarct size = (contralateral area-ipsilateral non-infarct area)/contralateral area $\times 100 \%$

\section{Quantitative Real-Time-Polymerase Chain Reaction (qPCR)}

Total RNA was extracted from the ischemic cerebral cortex using a commercial TRIzol kit, and RNA was then reverse-transcribed into cDNA using a PrimeScript RT reagent Kit(Vazyme,Nanjing,China).

Quantitative measurements were performed on an ABI 7500 PCR instrument (Applied Biosystems, USA) using a SYBR green kit(Applied Biosystems). Relative gene expression levels were normalized to GAPDH. The sequences of the primers used are as follows: TNF-a: (forward) TCGGTCCCAACAAGGAGGAG and (reverse) GGGTTGTCACTCGAGTTTTG; IL-1b: (forward) GCAACTGTTCCTGAACTCAACT and (reverse) ATCTTTTGGGGTCCGTCAACT; IL-6:(forward) GGCGGATCGGATGTTGTGAT and (reverse) GGACCCCAGACAATCGGTTG; GAPDH (forward) GCCAAGGCTGTGGGCAAGGT and (reverse) TCTCCAGGCGGCACGTCAGA.

\section{Western Blot Analysis}

Western blot analysis was then performed on the cerebral tissue within the ischemic areas. Proteins were homogenized in RIPA lysis buffer (Millipore, Billerica, MA, USA) containing protease inhibitors. The supernatant was assayed using a BCA Protein Assay Kit (Beyotime Biotech). The protein concentration was adjusted to the same concentration with protein buffer. The protein samples were then loaded, electrophoresis was performed, and the separated proteins were electrically transferred to polyvinylidene difluoride (PVDF) membrane. The membrane was blocked in PBS containing $0.1 \%$ Tween-20 and $5 \%$ nonfat milk for $2 \mathrm{~h}$ at room temperature. The membrane was then incubated with the primary antibodies overnight at $4^{\circ} \mathrm{C}$. anti-GAPDH(Bioworld), anti-Bax (Cell Signaling Technology), Anti-Bcl-2 (Bioworld), antiPhospho-IкBa(Cell Signaling Technology),anti-IкBa(Cell Signaling Technology)Danti-NF-кB (p-p65) (Cell Signaling Technology) $\square$ anti-NF-кB (p65) (Cell Signaling Technology) $\square$ anti-Phospho-p38 MAPK(Cell Signaling Technology) $\square$ anti-p38 MAPK(Cell Signaling Technology)Danti-Phospho-p44/42 MAPK (Erk1/2)

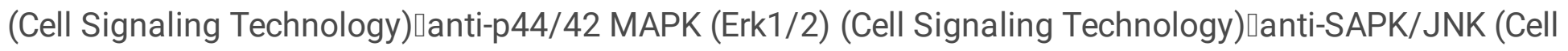
Signaling Technology), and anti-phospho-SAPK/JNK (Cell Signaling Technology)

After washing with PBST, the blots were incubated with HRP conjugated secondary antibody in blocking solution for 2 hand developed with the ECL chemiluminescence system(Thermo Company, West Chester, 
PA, USA). secondary antibody: Rabbit Anti-Goat IgG $(\mathrm{H}+\mathrm{L})-\mathrm{HRP}($ Bioworld),Mouse Anti-Goat $\lg \mathrm{G}(\mathrm{H}+\mathrm{L})$ AP(Bioworld).

\section{TUNEL Staining}

TUNEL staining was used to detect the apoptosis of neurons in the ischemic penumbra.TUNEL staining according to the manufacturer's instructions (Vazyme,Nanjing, China) .As previously described, the frozen sections were dried at $37^{\circ} \mathrm{C}$ for $2 \mathrm{~h}$, washed in PBS 3 times for 5 min each time, soaked in PBS containing $0.25 \%$ Triton X-100 for 15 min to permeabilize the cells, and washed with PBS 3 times. The cells were covered with $100 \mathrm{ml}$ of equilibrium buffer and incubated at room temperature for $5-10 \mathrm{~min}$. $50 \mathrm{ul}$ of terminal deoxynucleotidyl transferase recombinant(RTdT) incubation buffer was then added to each tissue slice, and the slices were then incubated for $60 \mathrm{~min}$ at $37^{\circ} \mathrm{C}$ in a dark humidified atmosphere. The slides were soaked in 2 X SSC solution, and the reaction was terminated after $15 \mathrm{~min}$. The cells were stained with DAPI for $15 \mathrm{~min}$ and then exposed to the quenchant. Laser scanning confocal microscopy was performed with a fluorescence microscope (Olympus X73). Positive cells were quantified via ImagePro Plus6.0 by blinded observers.

\section{Cytokine measurements}

Primary microglia with MVC (20nM) for $12 \mathrm{~h}$ after OGD/R. The supernatants were collected, and the concentrations of the cytokines TNF-a, IL-1 $\beta$ and IL- 6 were measured using enzyme-linked immunosorbent assays (ELISAs) according to the manufacturer's instructions (Cusabio Biotech, Wuhan, China)

\section{Statistics}

Relevant statistical analyses and graphing were performed using ImageJ and GraphPad Prism 8.0.2 All data are expressed as the mean \pm SEM. One-way analysis of variance (ANOVA) was used for multiple comparisons among groups, and Student's t-test was used for comparisons between two groups. $\mathrm{P}<0.05$ was considered significant.

\section{Results}

\section{MVC Reduced Infarct Volume and Improved Neurological Deficits After MCAO}

The molecular structure of MVC, 4,4-Difluoro-N-[(1S)-3-[(1R,5S)-3-(3-methyl-5-propan-2-yl-1,2,4-triazol-4yl)-8-azabicyclo[3.2.1]octan-8-yl]-1-phenylpropyl]cyclohexane-1-carboxamide, is shown in Fig. 1A., MVC treatment decreased the infarct volume $(P<0.05$, Fig. 1B). MVC treatment at higher doses $(20 \mathrm{umol} / \mathrm{kg})$ provided significant neuroprotection after $\mathrm{Cl} / \mathrm{R}$ injury $(\mathrm{P}<0.05$, Fig. $1 \mathrm{C})$. Because the $20 \mathrm{umol} / \mathrm{kg}$ dose produced the most significant effects, this dose was selected for future experiments. While the vehicle group exhibited poor neurological function as shown by the Longa and mNSS scores, MVC treatment (20umol/kg, i.p.) significantly alleviated the neurological deficits. Compared to the vehicle group, Longa 
and mNSS scores were significantly lower in the MVC treatment group $(P<0.05$, Fig. 1D, E), indicating that MVC treatment provided protective effects against $\mathrm{Cl} / \mathrm{R}$ injury in mice.

\section{MVC downregulates apoptosis-associated cytokines induced by $\mathrm{Cl} / \mathrm{R}$ injury and $\mathrm{OGD} / \mathrm{R}$}

Inhibiting the apoptotic effects of neurons in the ischemic penumbra is considered as a key target to protect against $\mathrm{Cl} / \mathrm{R}$ injury. Previous studies have shown that the change in $\mathrm{Bcl}-2 / \mathrm{Bax}$ ratio reflects the level of apoptosis[11]. .In this study, Western blot analysis of Bcl-2 and Bax was performed on brain tissue in mice at 3 days after MCAO. Consistent with the previous results, the expression ratio of Bcl2/Bax was decreased in the $\mathrm{Cl} / \mathrm{R}+$ vehicle group compared with those in the sham and MVC groups. However, MVC treatment resulted in an increase in $\mathrm{Bcl} 2 / \mathrm{Bax}$ ratio compared to the $\mathrm{Cl} / \mathrm{R}+$ vehicle group ( $\mathrm{P}$ $<0.05$, Fig. 2A, B) with increased Bcl-2 expression and decreased Bax expression. At $12 \mathrm{~h}$ after $O G D / R$, the western blots of microglia showed that the expression ratio of $\mathrm{Bcl}-2 / \mathrm{Bax}$ was decreased in the OGD/R group compared with those in the control and MVC groups $(P<0.05$, Fig. $2 C, D)$. TUNEL staining has also been used as an apoptotic marker. The number of TUNEL positive cells in the brain tissue was significantly decreased after MVC treatment compared to the $C I / R+$ vehicle group $(P<0.05$, Fig. $2 E, F)$. These results further support the anti-apoptotic effects of MVC in $\mathrm{Cl} / \mathrm{R}$.

\section{MVC Alleviated the Inflammatory Response After MCAO and OGD/R}

Inflammation has been demonstrated to be involved in all stages of $\mathrm{Cl} / \mathrm{R}$. To explore the potential antiinflammatory effects of MVC[12]., we detected the mRNA expression levels of pro-inflammatory cytokines, including TNFa, IL-1b and IL-6, by quantitative real-time polymerase chain reaction (qPCR). The mRNA levels of TNFa, IL-1b, and IL- 6 were all decreased after MVC treatment compared with the $\mathrm{Cl} / \mathrm{R}+$ vehicle group $(P<0.05$, Fig. $3 A-C)$. At $12 \mathrm{~h}$ after $O G D / R$, we evaluated the levels of the IL-1 $\beta, I L-6$ and TNF-a mRNAs and proteins using real-time PCR and ELISAs, respectively. Consistent with our hypothesis, MVC attenuated OGD-induced IL-1 $\beta$, IL-6 and TNF-a expression in microglia (Fig. 3D-I). These results indicate that MVC significantly alleviated the inflammatory response after $\mathrm{Cl} / \mathrm{R}$ injury and OGD/R.

\section{MVC Inhibited the NF-kB p65 Signalling}

After $\mathrm{Cl} / \mathrm{R}$ injury, nuclear factor-kappa B (NF-kB) pathway plays a central role in the post-ischemic proinflammatory response [13].. MVC has been reported to mitigate $\mathrm{Cl} / \mathrm{R}$ injury by inhibiting the NF-kB signlling pathway. In our study, the core proteins of the NF-kB signlling pathway were detected by Western blot. $\mathrm{Cl} / \mathrm{R}$ injury significantly increased the levels of phospho-IK $\beta / \mathrm{a}$ and phospho-p65 compared with the sham and MVC groups. However, MVC treatment downregulated phospho-lkBa and phospho-p65 levels, while upregulated IkBa levels compared with the $C I / R+$ vehicle group $(P<0.05$, Fig. 4A-D). At $12 \mathrm{~h}$ after $\mathrm{OGD} / \mathrm{R}$, significantly increased the levels of phospho-IK $\beta /$ a and phospho-p65 compared with the sham 
and MVC groups. MVC treatment downregulated phospho-lkBa and phospho-p65 levels, while upregulated IkBa levels compared with the OGD/R group $(P<0.05$, Fig. $4 \mathrm{E}-\mathrm{H})$. Taken together, these data indicated that MVC inhibits the NF-kB signaling pathway during $\mathrm{Cl} / \mathrm{R}$ injury and OGD/R.

\section{MVC suppressed the phosphorylation of ERK1/2, JNK and $\mathrm{P} 38$ in $\mathrm{Cl} / \mathrm{R}$ and $\mathrm{OGD} / \mathrm{R}$}

The MAPK signaling pathway is a well-known pathway involved in the inflammatory response that mainly consists of three MAPK subfamilies, including the ERK1/2, JNK and p38 families[14, 15].. Therefore, we aimed to investigate the molecular mechanism underlying the anti-inflammatory effect of $M V C$ in the $C l / R$ injury. As shown in Fig. $5 A-D, C l / R$ injury increased the levels of the phosphorylated ERK1/2, JNK, and p38 proteins, which was consistent with the results of our previous study. After MVC treatment, the phosphorylation of p38and JNK, but not ERK1/2, was suppressed (Fig. 5A-D). In addition, consistent with our previous findings, OGD/R increased the levels of the phosphorylated ERK $1 / 2, \mathrm{JNK}$, and p38 proteins. After MVC treatment, the phosphorylation of p38 and JNK, but not ERK1/2, was suppressed (Fig. 5E-I). According to these results, we concluded that MVC suppresses inflammation by inhhibiting the p38 and JNK MAPK signaling pathways without altering the activation of ERK1/2 MAPK.

The JNK signaling pathway activator AN reverses the neuroprotective effects of MVC on cerebral I/R injury and $O G D / R$.

To further investigate whether JNK pathway inhibition mediates the neuroprotective effects of MVC on cerebral I/R injury, we treated mice with the JNK signaling pathway activator AN ( $0.1 \mathrm{mg} / \mathrm{kg}$, i.p.) $30 \mathrm{~min}$ before ischemia. Western blot analysis showed that AN reversed the change in p-JNK levels induced by MVC treatment $(P<0.05$, Fig. 6A-B). For further verification, we evaluated the effects of different intervention methods on infarct volume in MCAO mice. TTC staining showed that compared with $I / R+$ MVC, AN treatment almost eliminated the protective effect of MVC after cerebral I/R injury (Fig. 7A-B). Based on the results above, the JNK pathway may contribute to the neuroprotective effects of MVC after cerebral I/R injury.

\section{Discussion}

Ischemic stroke is a serious vascular disease that significantly threats public health. Due to progressive improvements in recent years regarding our understanding of its pathogenesis, it is now recognized that cerebral ischemia-reperfusion injury is a complex pathophysiological process involving inflammation, cellular damage by oxygen-derived free radicals, energy depletion in the brain, intracellular calcium on overload, acidosis, toxicity of excitatory amino acids, nerve cell apoptosis, and the breakdown of the blood-brain barrier[16].. MVC has been reported to have many pharmacological effects, including antiinflammatory, and antiapoptotic effects[2].. Pharmacological research has demonstrated that MVC is capable of penetrating the blood brain barrier (BBB)[17].. MVC is a potent and selective antagonist of CCR5, which is localized on the surface of a variety of cells [18].. In the central nervous system, CCR5 is 
expressed by neurons, astrocytes and microglia[19].. In the present study, we observed the neuroprotective effect of MVC on the $\mathrm{Cl} / \mathrm{R}$ injury. Our results showed that MVC administration at 30 min, $24 \mathrm{~h}$ and $48 \mathrm{~h}$ after MCAO obviously attenuated the brain infarct size and neurological deficits on day 3 after $\mathrm{Cl} / \mathrm{R}$ injury. In the subsequent experiments, pre-treatment with MVC effectively inhibited the $\mathrm{Cl} / \mathrm{R}$ injury-induced proinflammatory mediators in mice and primary microglia by regulating the activation of the MAPK and NF-KB signaling pathways. According to our in vivo data, the i.p. injection of MVC attenuated the brain infarct volume and improved neurological deficits after transient focal ischemia. Furthermore, MVC significantly suppressed the release of inflammatory cytokines, suggesting that the protective effects of MVC on experimentally-induced stroke were at least partially mediated by the attenuation of microglia-mediated neuroinflammation. Taken together, MVC is a potential neuroprotective agent to treat ischemic brain injury. IL-1 $\beta$, IL- 6 and TNF- $\alpha$ are likely the most extensively studied proinflammatory cytokines involved in stroke. The levels of IL-1 $\beta$, IL- 6 and TNF- $\alpha$ are significantly increased in the brain within the first $24 \mathrm{~h}$ after the induction of focal cerebral ischemia in mice[20].. These three cytokines are mainly synthesized by microglia in the stroke-lesioned rodent brain [21].. In the present study, we confirmed that MVC not only suppresses the OGD-induced expression of the IL-1 $\beta$, IL- 6 and TNF-a mRNA and proteins in microglia, but also inhibits the production of these pro-inflammatory cytokines in the mouse brain after $\mathrm{Cl} / \mathrm{R}$ injury. In addition to MAPK pathways, NF-KB activation is also critically required for microglia-mediated CNS inflammation. $\mathrm{Cl} / \mathrm{R}$ injury significantly increased the levels of phospho-IKß/a and phospho-p65 compared with the sham and MVC groups. However, MVC treatment downregulated phospho-lkBa and phospho-p65 levels, while upregulated IkBa levels compared with the $\mathrm{Cl} / \mathrm{R}$ group. As shown in a recent study by Ganbold et al., NF-KB p65 silencing decreases the expression of pro-inflammatory cytokines and facilitates the anti-inflammatory polarization of microglia[22].. Consistent with these results, we unequivocally demonstrated that MVC pre-treatment significantly reduced phospho-lkBa and phospho-p65 levels, while upregulated IkBa levels compared with the OGD/R group. Subsequently, MVC suppressed NF-KB p65 activation and its nuclear translocation, suggesting that the IKB/NF-KB p65 signaling pathway is involved in the anti-inflammatory effects of MVC in OGD/R microglia. Several studies have reported that the MAPK signaling pathways, including the ERK1/2, JNK and p38 pathways, play vital roles in microglial activation and inflammatory response[14].. JNKs are essential mediators of microglia-mediated pro-inflammatory response[23]. .Thus, treatments that inhibit MAPKs may be a promising intervention for inflammatory responses induced by $\mathrm{Cl} / \mathrm{R}$ injury. In our current study, we confirmed that these three MAPK signaling pathways that were involved in that phosphorylation of p38 and JNK were markedly diminished in $\mathrm{Cl} / \mathrm{R}$ injury after MVC pre-treatment, suggesting that $\mathrm{p} 38$ and JNK are the two important molecular targets of MVC. Furthermore, MVC tended to decrease the levels of phospho-JNK. In this study, we provided robust evidence that the inhibition of JNK activation can mediate the neuroprotective effects of MVC in a cerebral I/R injury model. To confirm the above conclusion, a JNK signaling pathway agonist (AN) was employed in the MCAO model. AN significantly increased the level of p-JNK and reversed the protective effects of MVC on cerebral I/R injury. AN-induced excessive activation of JNK may explain the finding that AN almost completely blocked the neuroprotective effects of MVC. Nevertheless, to reduce the use of experimental animals, the number of mice in each group was relatively small, which may cause potential bias. Further experiments will be 
conducted to validate the effect of MVC on mice models of ischemic stroke to make our research more meaningful and intriguing.

\section{Declarations}

Ethics Approval and Consent to Participate All animal testing procedures were approved by the local experimental ethics committee and were performed in accordance with the guidelines of the National Institutes of Health Guide for the Care and Use of Laboratory Animals. All experimental procedures were approved by the Animal Ethics Committee of Yangzhou University (YZUNSFC2020-LCYXY-136).

Conflict of Interest The authors declare no competing interests.

AcknowledgmentsThe authors would like to acknowledge Xiang Cao, Affiliated of Drum Tower Hospital, Medical School of Nanjing University, China.

\section{References}

1. Hurford, R., A. Sekhar, T. A. T. Hughes, and K. W. Muir. 2020. Diagnosis and Management of Acute Ischaemic Stroke. Pract Neurol. https://dx.doi.org/10.1136/practneurol-2020-002557.

2. Cipriani, S., D. Francisci, A. Mencarelli, B. Renga, E. Schiaroli, C. D'Amore, F. Baldelli, and S. Fiorucci. 2013. Efficacy of the Ccr5 Antagonist Maraviroc in Reducing Early, Ritonavir-Induced Atherogenesis and Advanced Plaque Progression in Mice. Circulation. https://dx.doi.org/10.1161/circulationaha.113.001278.

3. Liu, F. C., C. W. Zheng, and H. P. Yu. 2016. Maraviroc-Mediated Lung Protection Following TraumaHemorrhagic Shock. Biomed Res Int. https://dx.doi.org/10.1155/2016/5302069.

4. Kraft-Terry, S. D., A. R. Stothert, S. Buch, and H. E. Gendelman. 2010. Hiv-1 Neuroimmunity in the Era of Antiretroviral Therapy. Neurobiol Dis. https://dx.doi.org/10.1016/j.nbd.2009.12.015.

5. Escola, J. M., G. Kuenzi, H. Gaertner, M. Foti, and O. Hartley. 2010. Cc Chemokine Receptor 5 (Ccr5) Desensitization: Cycling Receptors Accumulate in the Trans-Golgi Network. J Biol Chem. https://dx.doi.org/10.1074/jbc.M110.153460.

6. Venuti, A., C. Pastori, and L. Lopalco. 2017. The Role of Natural Antibodies to Cc Chemokine Receptor 5 in Hiv Infection. Front Immunol. https://dx.doi.org/10.3389/fimmu.2017.01358.

7. Akahori, T., M. Sho, H. Kashizuka, T. Nomi, H. Kanehiro, and Y. Nakajima. 2006. A Novel Ccr5/Cxcr3 Antagonist Protects Intestinal Ischemia/Reperfusion Injury. Transplant Proc. https://dx.doi.org/10.1016/j.transproceed.2006.10.115.

8. Grommes, J., M. Drechsler, and O. Soehnlein. 2014. Ccr5 and Fpr1 Mediate Neutrophil Recruitment in Endotoxin-Induced Lung Injury. J Innate Immun. https://dx.doi.org/10.1159/000353229.

9. Chiang, T., R. O. Messing, and W. H. Chou. 2011. Mouse Model of Middle Cerebral Artery Occlusion. J Vis Exp. https://dx.doi.org/10.3791/2761. 
10. Yu, H., L. Song, X. Cao, W. Li, Y. Zhao, J. Chen, J. Li, Y. Chen, W. Yu, and Y. Xu. 2020. Hederagenin Attenuates Cerebral Ischaemia/Reperfusion Injury by Regulating Mlk3 Signalling. Front Pharmacol. https://dx.doi.org/10.3389/fphar.2020.01173.

11. Cong, C., L. Kluwe, S. Li, X. Liu, Y. Liu, H. Liu, W. Gui, T. Liu, and L. Xu. 2019. Paeoniflorin Inhibits Tributyltin Chloride-Induced Apoptosis in Hypothalamic Neurons Via Inhibition of Mkk4-Jnk Signaling Pathway. J Ethnopharmacol. https://dx.doi.org/10.1016/j.jep.2019.03.030.

12. Karampoor, S., H. Zahednasab, R. Amini, M. Esghaei, M. Sholeh, and H. Keyvani. 2020. Maraviroc Attenuates the Pathogenesis of Experimental Autoimmune Encephalitis. Int Immunopharmacol. https://dx.doi.org/10.1016/j.intimp.2019.106138.

13. Zhang, D. D., M. J. Zou, Y. T. Zhang, W. L. Fu, T. Xu, J. X. Wang, W. R. Xia, Z. G. Huang, X. D. Gan, X. M. Zhu, and D. G. Xu. 2017. A Novel Il-1ra-Pep Fusion Protein with Enhanced Brain Penetration Ameliorates Cerebral Ischemia-Reperfusion Injury by Inhibition of Oxidative Stress and Neuroinflammation. Exp Neurol. https://dx.doi.org/10.1016/j.expneurol.2017.06.012.

14. Ji, R. R., R. W. th Gereau, M. Malcangio, and G. R. Strichartz. 2009. Map Kinase and Pain. Brain Res Rev. https://dx.doi.org/10.1016/j.brainresrev.2008.12.011.

15. Piotrowska, A., K. Kwiatkowski, E. Rojewska, W. Makuch, and J. Mika. 2016. Maraviroc Reduces Neuropathic Pain through Polarization of Microglia and Astroglia - Evidence from In vivo and In vitro Studies. Neuropharmacology. https://dx.doi.org/10.1016/j.neuropharm.2016.04.024.

16. Jin, R., G. Yang, and G. Li. 2010. Inflammatory Mechanisms in Ischemic Stroke: Role of Inflammatory Cells. J Leukoc Biol. https://dx.doi.org/10.1189/jlb.1109766.

17. Haruwaka, K., A. Ikegami, Y. Tachibana, N. Ohno, H. Konishi, A. Hashimoto, M. Matsumoto, D. Kato, R. Ono, H. Kiyama, A. J. Moorhouse, J. Nabekura, and H. Wake. 2019. Dual Microglia Effects on Blood Brain Barrier Permeability Induced by Systemic Inflammation. Nat Commun. https://dx.doi.org/10.1038/s41467-019-13812-z.

18. Levy, J. A. 2009. Hiv Pathogenesis: 25 Years of Progress and Persistent Challenges. Aids. https://dx.doi.org/10.1097/QAD.0b013e3283217f9f.

19. Kaul, M., Q. Ma, K. E. Medders, M. K. Desai, and S. A. Lipton. 2007. Hiv-1 Coreceptors Ccr5 and Cxcr4 Both Mediate Neuronal Cell Death but Ccr5 Paradoxically Can Also Contribute to Protection. Cell Death Differ. https://dx.doi.org/10.1038/sj.cdd.4402006.

20. Hill, J. K., L. Gunion-Rinker, D. Kulhanek, N. Lessov, S. Kim, W. M. Clark, M. P. Dixon, R. Nishi, M. P. Stenzel-Poore, and F. P. Eckenstein. 1999. Temporal Modulation of Cytokine Expression Following Focal Cerebral Ischemia in Mice. Brain Res. https://dx.doi.org/10.1016/s0006-8993(98)01140-8.

21. Lambertsen, K. L., K. Biber, and B. Finsen. 2012. Inflammatory Cytokines in Experimental and Human Stroke. J Cereb Blood Flow Metab. https://dx.doi.org/10.1038/jcbfm.2012.88.

22. Ganbold, T., Q. Bao, J. Zandan, A. Hasi, and H. Baigude. 2020. Modulation of Microglia Polarization through Silencing of Nf-Kb P65 by Functionalized Curdlan Nanoparticle-Mediated Rnai. ACS App/ Mater Interfaces. https://dx.doi.org/10.1021/acsami.9b23004. 
23. Waetzig, V., K. Czeloth, U. Hidding, K. Mielke, M. Kanzow, S. Brecht, M. Goetz, R. Lucius, and T. Herdegen, and U. K. Hanisch. 2005. C-Jun N-Terminal Kinases (Jnks) Mediate Pro-Inflammatory Actions of Microglia. Glia. https://dx.doi.org/10.1002/glia.20173.

24. Ligends.

\section{Figures}

\section{Fig1}

A

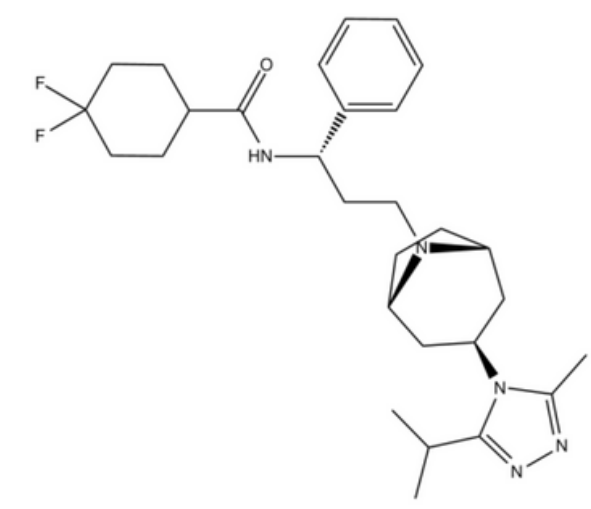

D

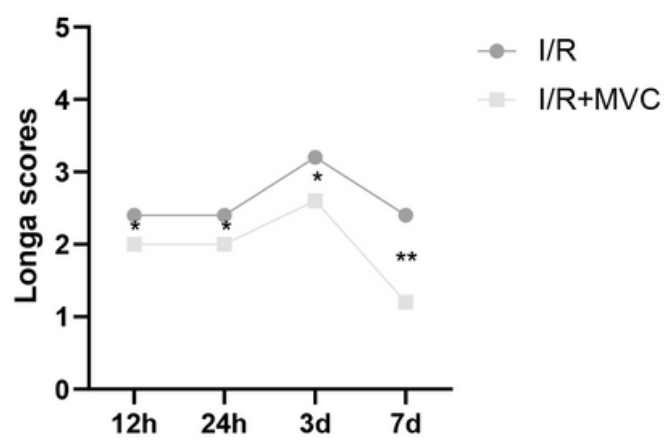

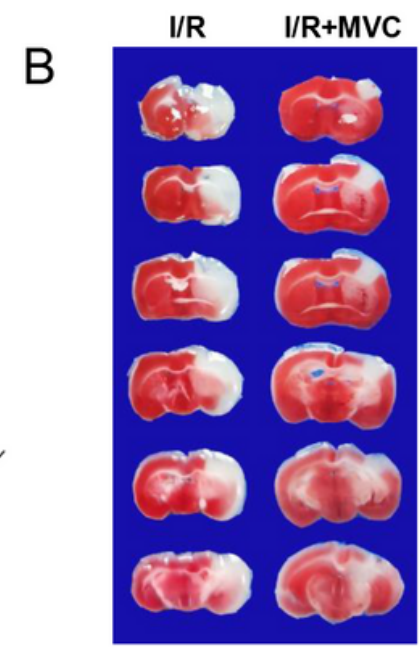

E
C
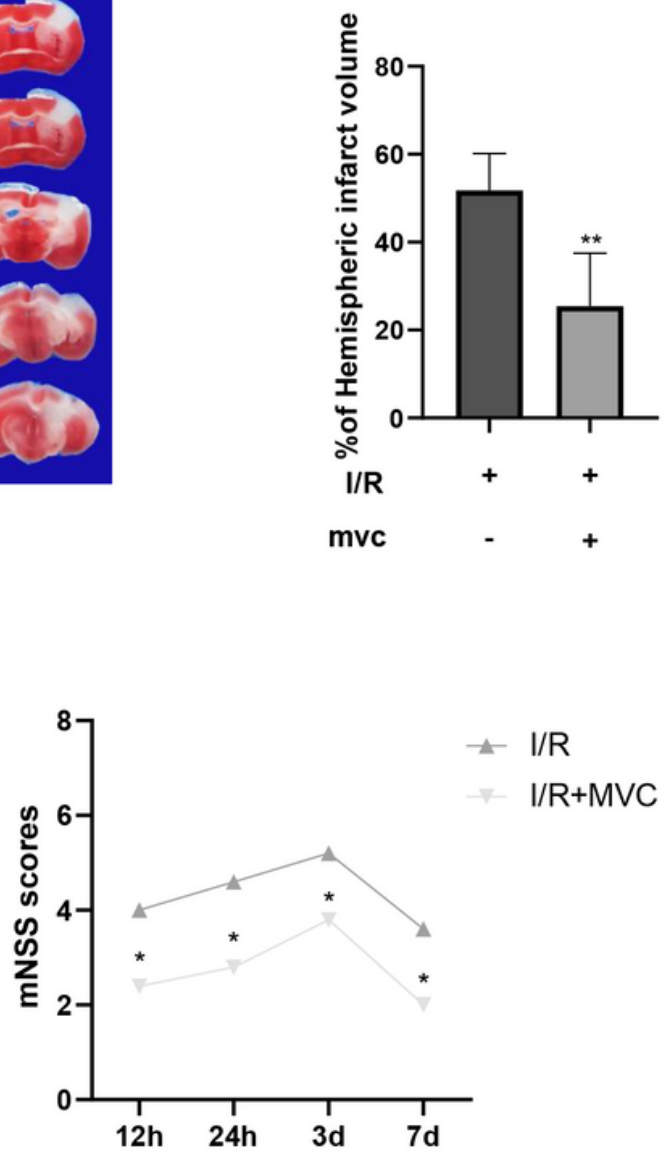

\section{Figure 1}

MVC reduced infarct volumes and improved neurological defificits after MCAO. The mice were injected with MVC vehicle (i.p.) once daily for 3 consecutive days after MCAO.Primary microglia with MVC (20nM) for $12 \mathrm{~h}$ after OGD/R.(A) The molecular structure of MVC is shown. (B) Mice were injected with MVC at 
doses of $20 \mathrm{mg} / \mathrm{kg}$ (i.p.) after MCAO. The representative TTC-stained coronal sections in vehicle- and MVC-treated mice are shown. (C) MVC signifificantly reduced infarct volumes after MCAO. MVC treatments signifificantly reduced Longa (D) and mNSS (E) scores after MCAO. The bar graphs represent the mean \pm SEM of 6 brains in each group. ${ }^{*} P<0.05$ versus the MCAO group

Fig2

A

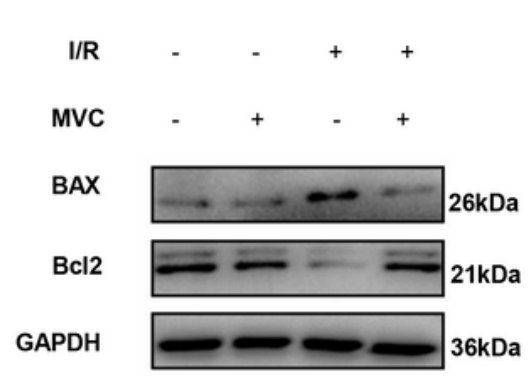

C

E

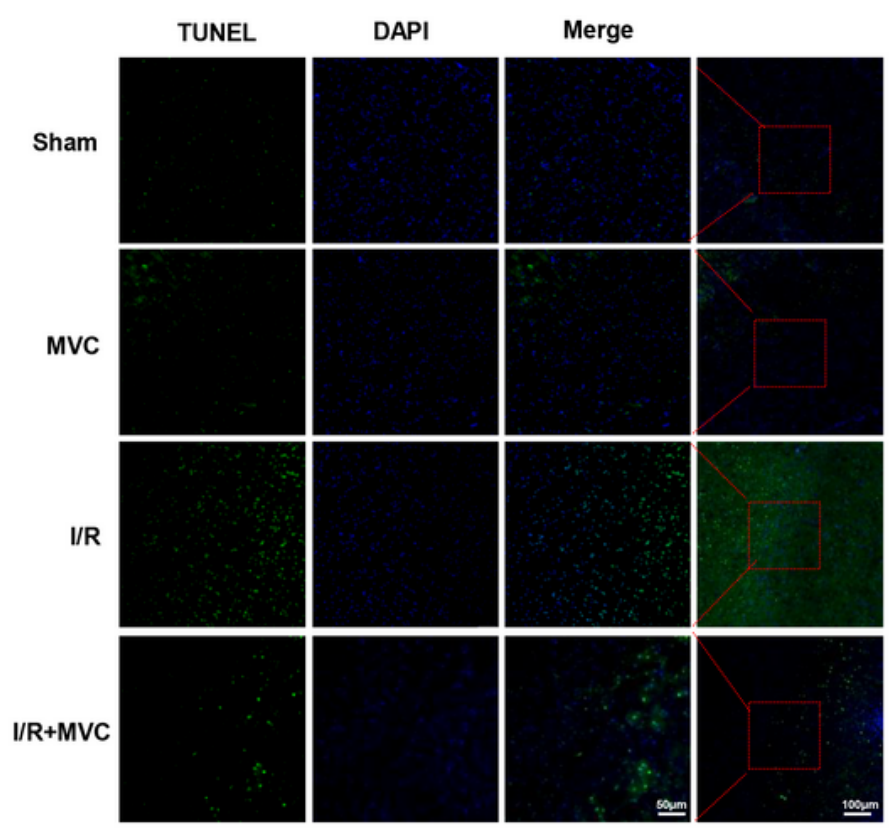

B
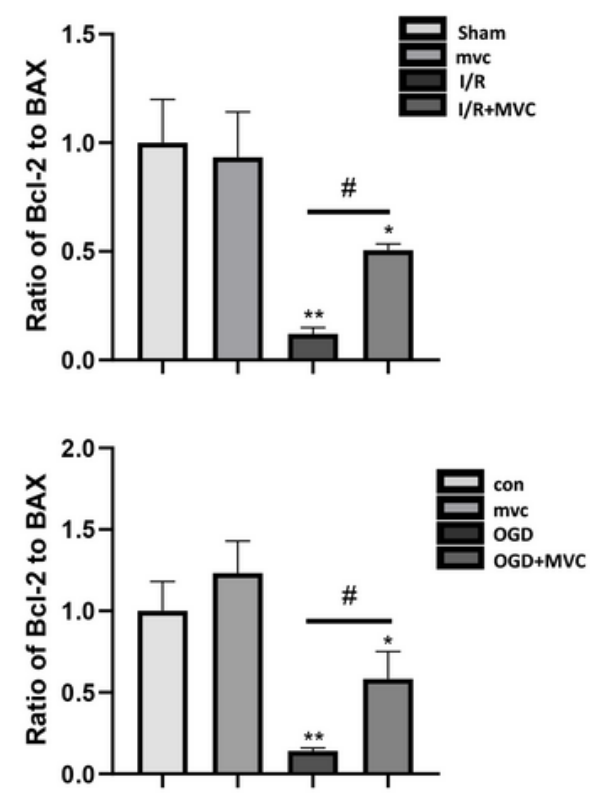

$\mathrm{F}$

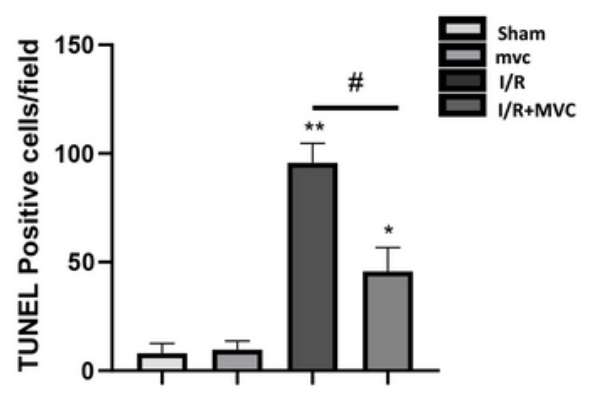

Figure 2 
MVC downregulates apoptosis-associated cytokines induced by $\mathrm{Cl} / \mathrm{R}$ injury and OGD/R. Mice were treated with MVC $(20 \mathrm{mg} / \mathrm{kg})$ or vehicle (i.p.) once daily for 3 consecutive days after MCAO. Primary microglia with MVC (20nM) for $12 \mathrm{~h}$ after OGD/R.Western blot was used to detect the expression levels of apoptosis-related proteins, and TUNEL staining was used to measure the apoptotic levels after $\mathrm{Cl} / \mathrm{R}$ injury. A At 3 days after MCAO, Representative images show the expression levels of $\mathrm{Bcl}-2$, Bax by Western Blot analysis with GAPDH as a loading control. B Quantitative analysis of the Bcl-2 to Bax ratio. $\mathrm{C}$ At $12 \mathrm{~h}$ after OGD/R,Representative images show the expression levels of Bcl-2, Bax by Western Blot analysis with GAPDH as a loading control. D Quantitative analysis of the Bcl-2 to Bax ratio. E TUNELpositive cells showed green fluorescence, and DAPI-positive cells showed blue fluorescence. $F$ Quantifification of the number of TUNEL-positive neural cells in each group. MVC treatment signifificantly decreased the number of TUNEL-positive neural cells after $\mathrm{Cl} / \mathrm{R}$ injury. Scale bar, $50 \mu \mathrm{m}$; magnifification, 20x. The bar graphs represent the mean \pm SEM of three brains in each group. ${ }^{*} \mathrm{P}<0.05$ versus the $\mathrm{Cl} / \mathrm{R}$ group. 
Fig3
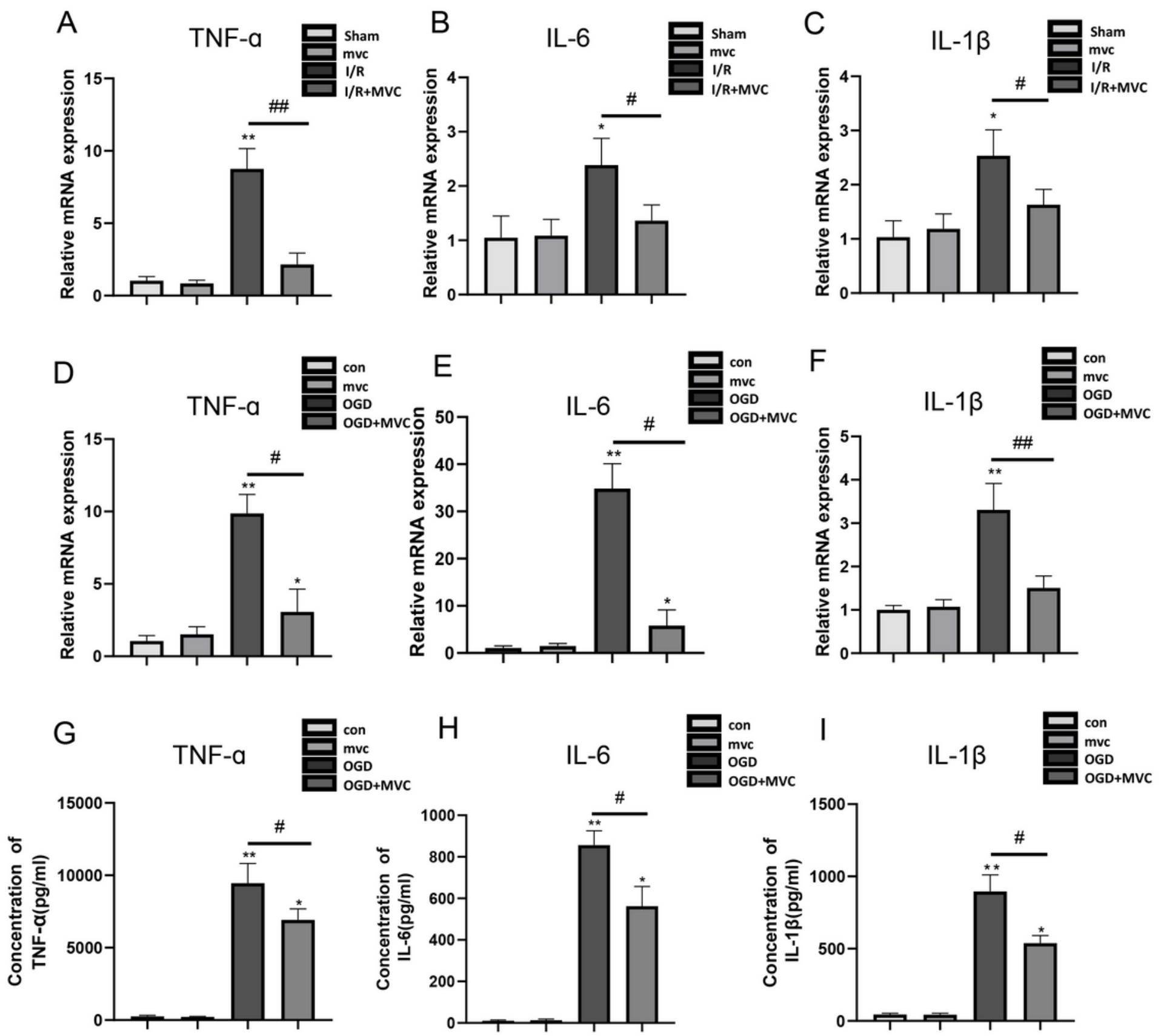

\section{Figure 3}

MVC Alleviated the Inflammatory Response After MCAO and OGD/R.The mice were treated with MVC (20 $\mathrm{mg} / \mathrm{kg}$, i.p.) or vehicle once daily for 3 consecutive days after $\mathrm{Cl} / \mathrm{R}$ injury. Primary microglia with MVC $(20 \mathrm{nM})$ for $12 \mathrm{~h}$ after OGD/R.Pro-inflflammatory cytokine levels were determined by qRT-PCR, ELISA. A-C The mRNA levels of TNF-a, IL-6, and IL-1b in the ischaemic penumbra tissues. MVC signifificantly decreased the mRNA levels of TNF-a, IL-6, and IL-1b after Cl/R injury. D-F The mRNA levels of TNF-a, IL-6, 
and IL-1b in microglia.MVC signifificantly decreased the mRNA levels of TNF-a, IL-6, and IL-1b after OGD/R. G-I the protein levels of TNF-a, IL-6 and IL-1b were evaluated by ELISA.MVC signifificantly decreased the protein levels of TNF-a, IL-6, and IL-1b after OGD/R.

\section{Fig4}

A

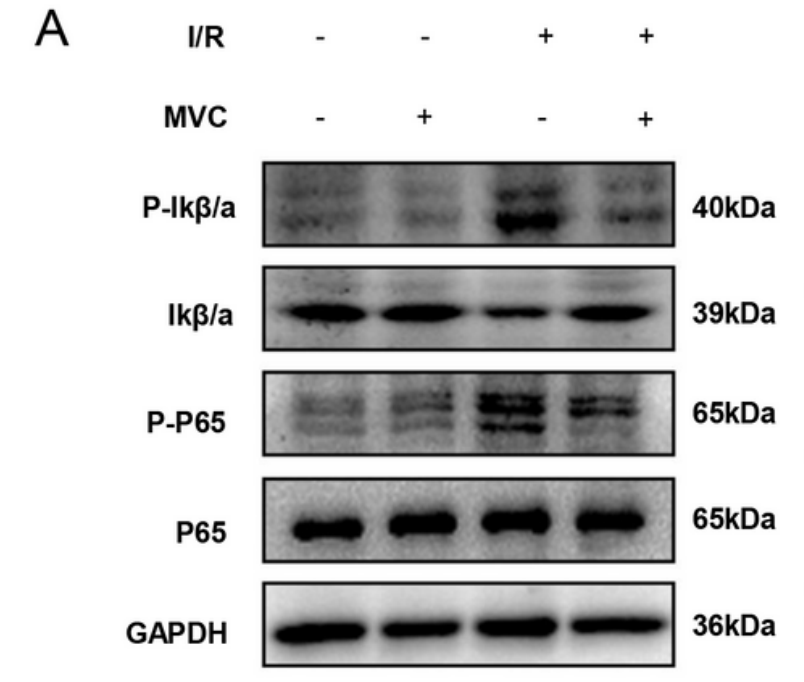

B

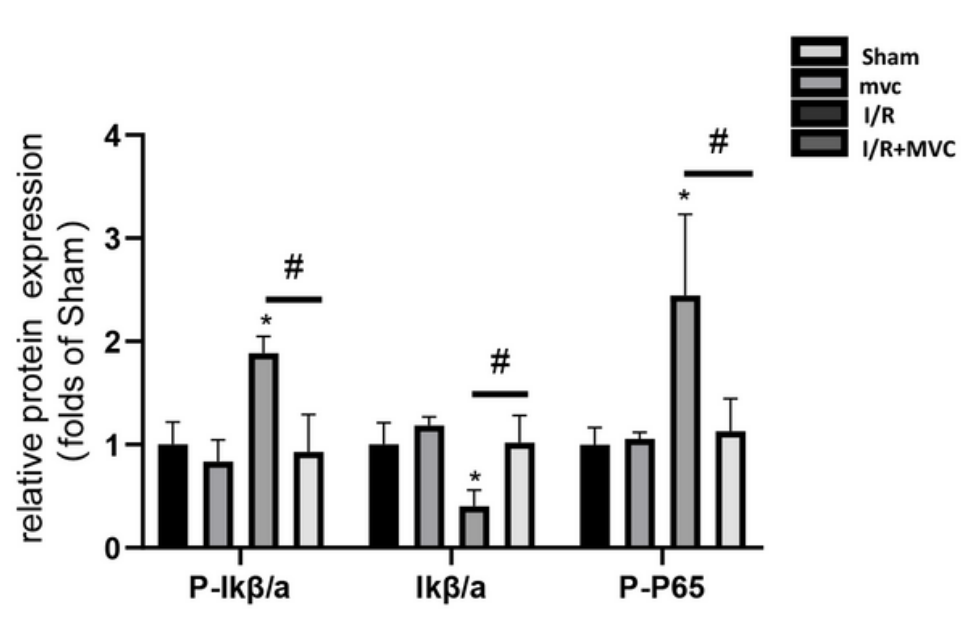

C
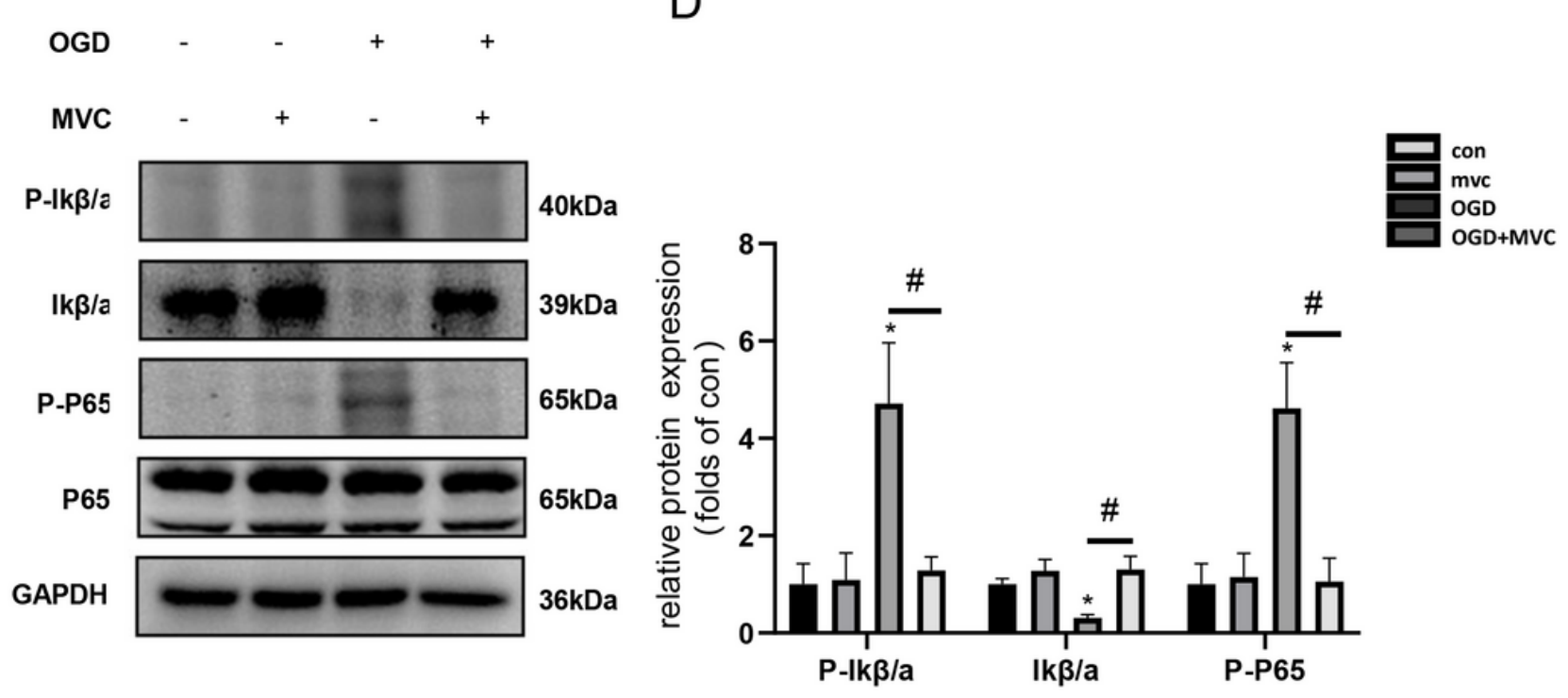

Figure 4

MVC Inhibited the NF-kB p65 Signalling.The mice were treated with MVC $(20 \mathrm{mmol} / \mathrm{kg}$, i.p.) or vehicle once daily for 3 consecutive days after $\mathrm{Cl} / \mathrm{R}$. Primary microglia with MVC (20nM) for $12 \mathrm{~h}$ after OGD/R.The protein levels were detected by Western blot. A The expression of p-IKBa, IkBa, p65 and p-p65 in the ischaemic penumbra tissues was analyzed by Western blotting. GAPDH was used as a loading control. B-D The greyscale value of each band was evaluated by ImageJ software.Quantitative analysis 
showed that MVC decreased the expression levels of $\mathrm{p}-\mathrm{I} \mathrm{KBa}, \mathrm{I} \mathrm{KBa}$ and $\mathrm{p}-\mathrm{p} 65$ after $\mathrm{Cl} / \mathrm{R}$ injury. E The expression of p-ІкBa, IкBa, p65 and p-p65 in microglia was analyzed by Western blotting. GAPDH was used as a loading control. G-H Quantitative analysis showed that MVC decreased the expression levels of $\mathrm{p}-\mathrm{IKBa}_{\mathrm{K}} \mathrm{IKBa}_{\mathrm{K}}$ and $\mathrm{p}-\mathrm{p} 65$ after OGD/R.The bar graphs represent the mean $\pm \mathrm{SEM}$ of three brains in each group. ${ }^{*} \mathrm{P}<0.05$ versus the $\mathrm{Cl} / \mathrm{R}$ group.

\section{Fig5}

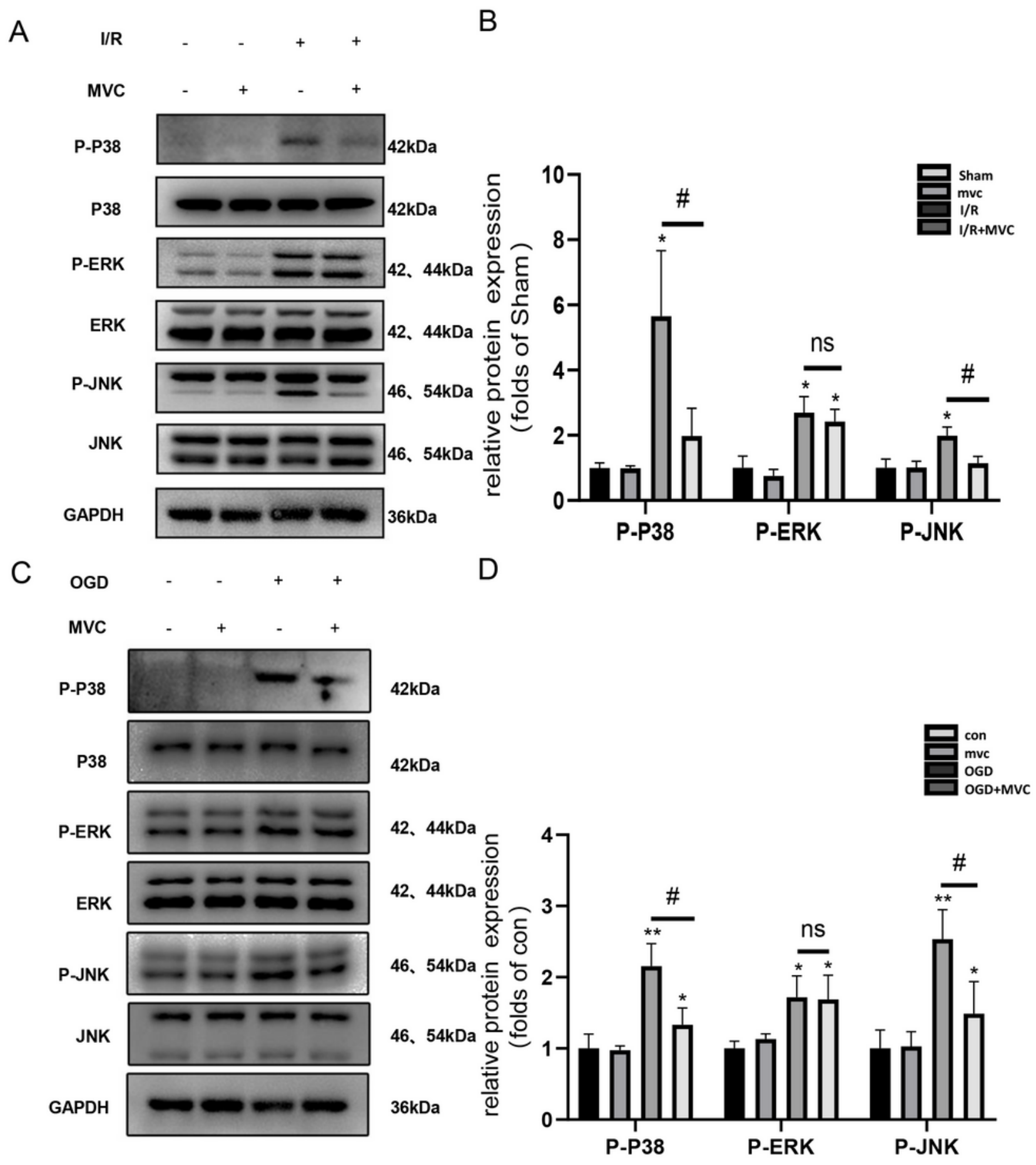

Figure 5 
MVC suppressed the phosphorylation of ERK1/2, JNK and P38 in CI/R and OGD/R.Mice were treated with MVC ( $20 \mathrm{mg} / \mathrm{kg}$, i.p.) or vehicle once daily for 3 consecutive days after $\mathrm{Cl} / \mathrm{R}$.Primary microglia with MVC $(20 \mathrm{nM})$ for $12 \mathrm{~h}$ after OGD/R. After 3 days of reperfusion, cerebral tissue was collected, and protein levels were detected by Western blot. A The representative images show the levels of $p$-p38, p38, p-ERK, ERK, pJNK, JNK, and GAPDH. GAPDH was used as a loading control. B Quantitative analysis showed that MVC decreased the expression level of p-p38 and p-JNK, but not that of p-ERK, after $\mathrm{Cl} / \mathrm{R}$ injury. C The representative images show the levels of p-p38, p38, p-ERK, ERK, p-JNK, JNK, and GAPDH. GAPDH was used as a loading control. D Quantitative analysis showed that MVC decreased the expression level of $p$ p38 and p-JNK, but not that of $p$-ERK, after OGD/R. The bar graphs represent the mean \pm SEM of three brains in each group. ${ }^{*} \mathrm{P}<0.05$ versus the $\mathrm{Cl} / \mathrm{R}$ group and $\mathrm{OGD} / \mathrm{R}$ group.

\section{Fig6}

A

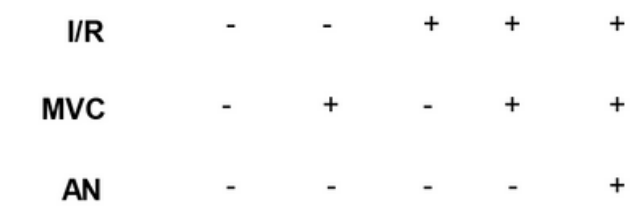

B

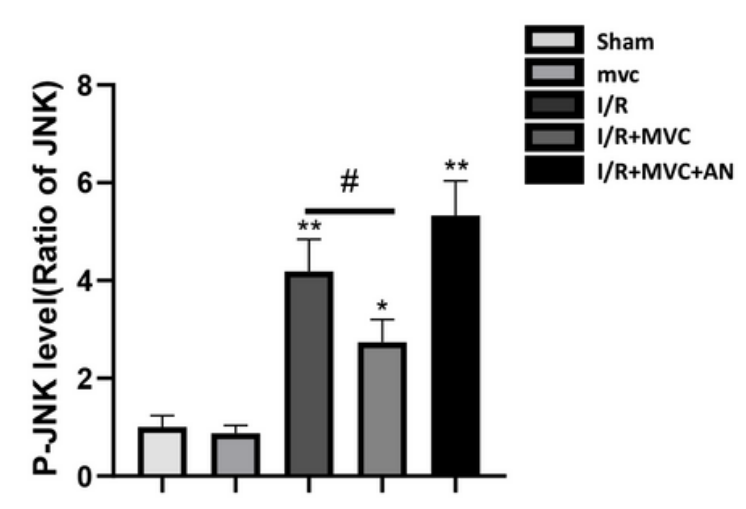

C

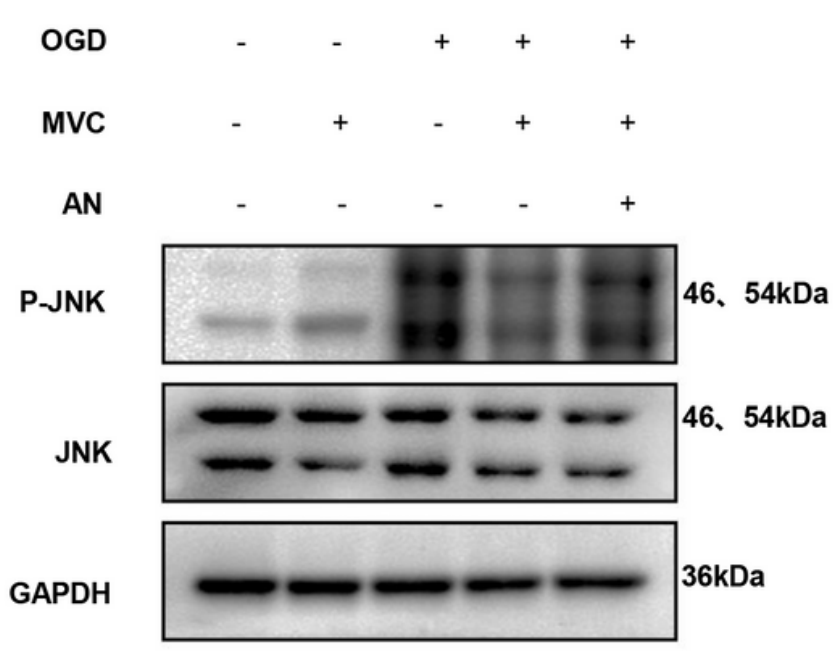

D

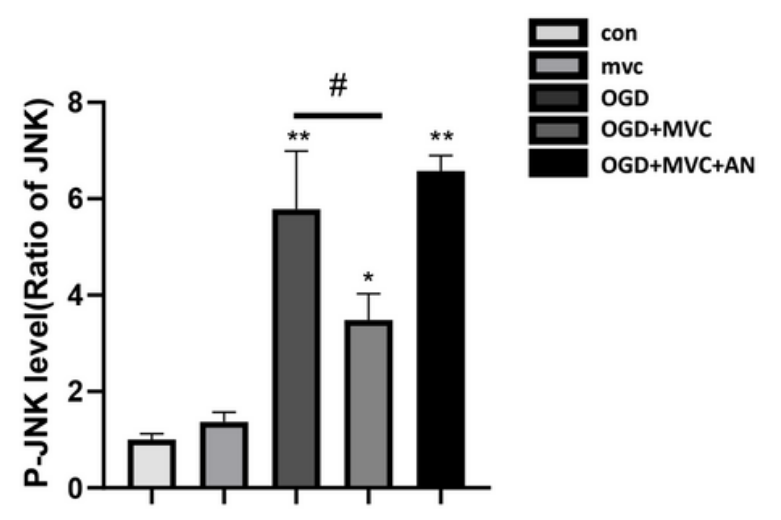

Figure 6 
The JNK signaling pathway activator AN reverses the neuroprotective effects of MVC on cerebral I/R injury and OGD/R.Mice were treated with MVC $(20 \mathrm{mg} / \mathrm{kg}$, i.p.) or vehicle once daily for 3 consecutive days after $\mathrm{Cl} / \mathrm{R}$.Primary microglia with MVC (20nM) for $12 \mathrm{~h}$ after OGD/R. Protein levels were determined by Western blot. A, B Mice were treated with MVC $(20 \mathrm{mg} / \mathrm{kg}$, i.p.) or vehicle $1 \mathrm{~h}$ after ischemia and AN $(0.1 \mathrm{mg} / \mathrm{kg}$, i.p.) was given $30 \mathrm{~min}$ before ischemia. Western blot and quantitative analysis showed that AN treatment reversed the inhibitory effect of MVC on JNK phosphorylation.C, D Primary microglia with MVC (20nM) after OGD/R and AN (0.1nM) was given 30 min before OGD.Western blot and quantitative analysis showed that AN treatment reversed the inhibitory effect of MVC on JNK phosphorylation.

\section{Fig7}
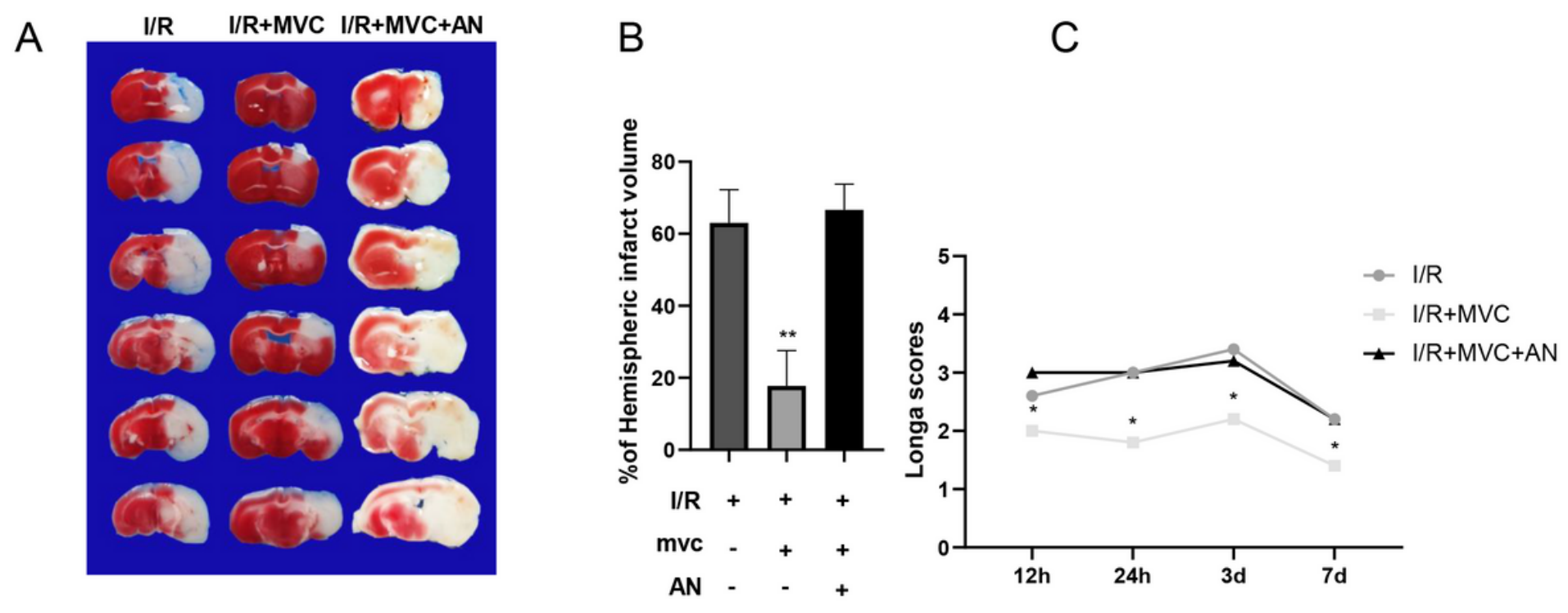

D

E

$\mathrm{F}$
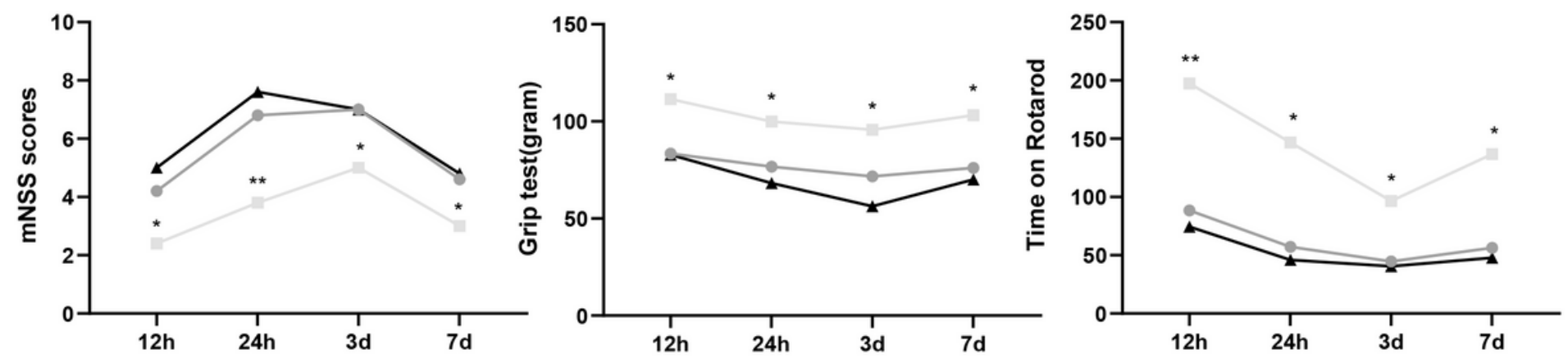

\section{Figure 7}

A The volume of cerebral infarction was measured by TTC staining. B Statistical analysis of representative TTC-stained coronal sections indicated that the JNK agonist AN almost blocked the protective effect of MVC. The bar graph showed the mean \pm S.E.M. of three brains in each group, *, 
indicating $\mathrm{P}<0.05$ compared with the I/R group. C-F MVC treatments signifificantly reduced Longa scores,mNSS scores,Grip test and Time on Rotarod after $\mathrm{Cl} / \mathrm{R}$ injury, but the JNK agonist AN almost blocked the protective effect of MVC. 